\title{
Neutrino oscillations: present status and outlook ${ }^{1}$
}

\author{
Thomas Schwetz \\ Physics Department, Theory Division, CERN, CH-1211 Geneva 23, Switzerland
}

\begin{abstract}
I summarize the status of three-flavour neutrino oscillations with date of Oct. 2007, and provide an outlook for the developments to be expected in the near future. Furthermore, I discuss the status of sterile neutrino oscillation interpretations of the LSND anomaly in the light of recent MiniBooNE results, and comment on implications for the future neutrino oscillation program.
\end{abstract}

Keywords: Neutrino oscillations

PACS: $14.60 . \mathrm{Lm}, 14.60 . \mathrm{Pq}, 14.60 . \mathrm{St}$

Thanks to the spectacular developments in neutrino oscillation experiments in the last years we have now a rough picture of the parameters governing three-flavour oscillations (see also Ref. [1] for an overview): There are two mass-squared differences separated roughly by a factor 30 , there are two large mixing angles $\left(\theta_{23}\right.$, which could even be 45 , and $\theta_{12}$, which is large but smaller than 45 at very high significance), and one mixing angle which has to be small $\left(\theta_{13}\right)$. Present data is consistent with two possibilities for the neutrino mass ordering, conventionally parametrized by the sign of $\Delta m_{31}^{2}$ : In the normal ordering $\left(\Delta m_{31}^{2}>0\right)$ the mass state which contains predominantly the electron neutrino has the smallest mass, whereas in the inverted ordering $\left(\Delta m_{31}^{2}<0\right)$ it is part of a nearly degenerate doublet of mass states which is separated from the lightest neutrino mass by $j \Delta m_{31}^{2} j$

\section{GLOBAL THREE-FLAVOUR ANALYSIS}

In this section I present an update on the determination of three-neutrino oscillation parameters from a global analysis of latest world neutrino oscillation data from solar, atmospheric, reactor, and accelerator experiments. These results are based on work in collaboration with M. Maltoni, M. Tortola and J.W.F. Valle, published in Refs. [2, 3] (see also the arXiv version 6 of Ref. [3] for updated results). The present determination of the threeflavour oscillation parameters is summarized in Tab. 1 where the best fit points and the $2 \sigma$ and $3 \sigma$ allowed ranges are given.

\footnotetext{
${ }^{1}$ Plenarly talk at NuFact07, Okayama, Japan.
}

TABLE 1. Best-fit values, $2 \sigma$ and $3 \sigma$ intervals (1 d.o.f.) for the three-flavour neutrino oscillation parameters from global data including solar, atmospheric, reactor (KamLAND and CHOOZ) and accelerator (K2K and MINOS) experiments.

\begin{tabular}{llcc}
\hline Parameter & Best fit & $\mathbf{2} \sigma$ & $\mathbf{3} \sigma$ \\
\hline$\Delta m_{21}^{2}\left[10^{5} \mathrm{eV}^{2}\right]$ & 7.6 & $7.3-8.1$ & $7.1-8.3$ \\
$\Delta m_{31}^{2} j\left[10^{3} \mathrm{eV}^{2}\right]$ & 2.4 & $2.1-2.7$ & $2.0-2.8$ \\
$\sin ^{2} \theta_{12}$ & 0.32 & $0.28-0.37$ & $0.26-0.40$ \\
$\sin ^{2} \theta_{23}$ & 0.50 & $0.38-0.63$ & $0.34-0.67$ \\
$\sin ^{2} \theta_{13}$ & 0.007 & 0.033 & 0.050 \\
\hline
\end{tabular}

This analysis includes new data released this summer by the MINOS [4, 5] and KamLAND [6, 7] collaborations which lead to an improved determination of the mass-squared differences $j \Delta m_{31}^{2}$ jand $\Delta m_{21}^{2}$, respectively, mainly due to the precise spectral information. New MINOS data have been collected from June 2006 to July 2007 (Run-IIa), and they have been analyzed together with the first data sample (Run-I), with a total exposure of $2.510^{20}$ p.o.t. In total, $563 v_{\mu}$ events have been observed at the far detector, while $738 \quad 30$ events were expected for no oscillation. KamLAND data presented at TAUP2007 [6] correspond to a total exposure of 2881 ton year, almost 4 times larger than 2004 data 7$]$. Apart from the increased statistics also systematic uncertainties have been improved: Thanks to the full volume calibration the error on the fiducial mass has been reduced from $4.7 \%$ to $1.8 \%$. The main limitation for the $\Delta m_{21}^{2}$ measurement comes now from the uncertainty on the energy scale of $1.5 \%$. Details of our KamLAND analysis are described in appendix A of Ref. [3]. We use the data binned in equal bins in $1=E$ to make optimal use of spectral in- 

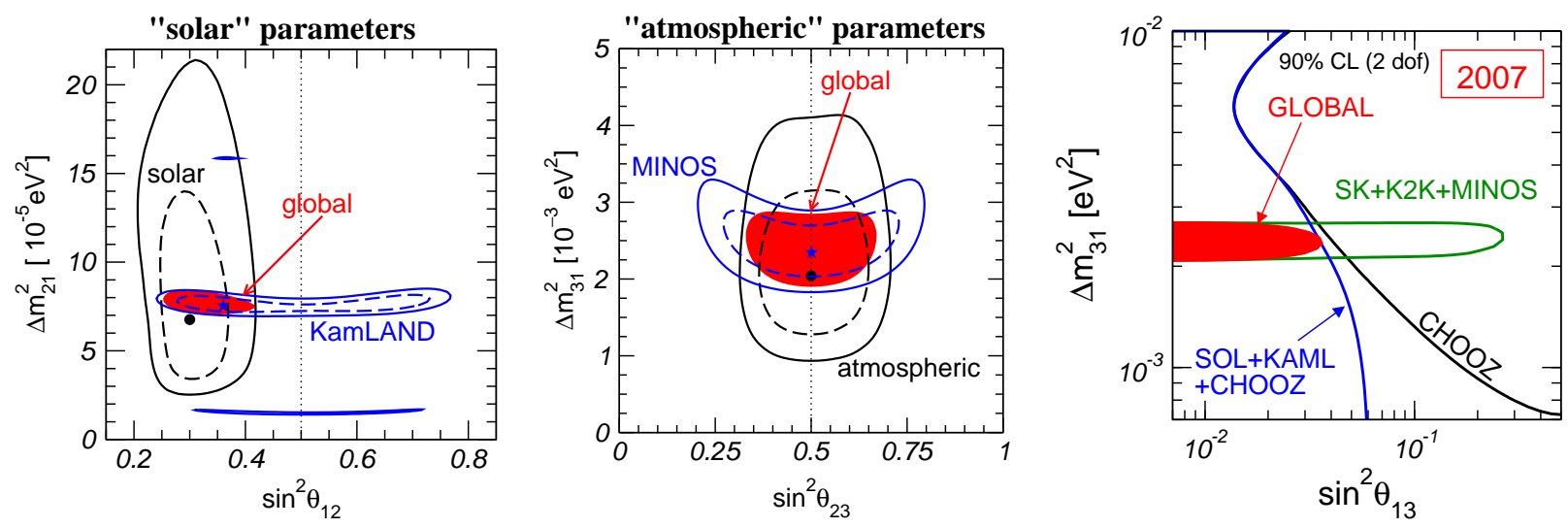

FIGURE 1. Determination of the leading "solar" (left) and "atmospheric" (middle) oscillation parameters from the interplay of data from artificial and natural neutrino sources. Right: Constraint on $\theta_{13}$ from the global analysis of neutrino data.

formation, we take into account the (small) matter effect and carefully include various systematics. As previously we restrict the analysis to the prompt energy range above $2.6 \mathrm{MeV}$ to avoid large contributions from geoneutrinos and backgrounds. In that energy range 1549 reactor neutrinos events and a background of 63 events are expected without oscillations, whereas the observed number of events is 985 .

Fig. 1 illustrates how the determination of the leading "solar" $\left(\theta_{12}\right.$ and $\left.\Delta m_{21}^{2}\right)$ and "atmospheric" $\left(\theta_{23}\right.$ and $j \Delta m_{31}^{2}$ ) oscillation parameters emerges from the complementarity of data from natural (sun and atmosphere) and men-made (reactor and accelerator) neutrino sources. Spectral information from KamLAND data leads to an accurate determination of $\Delta m_{21}^{2}$ with the remarkable precision of $5 \%$ at $2 \sigma$. KamLAND data start also to contribute to the lower bound on $\sin ^{2} \theta_{12}$, whereas the upper bound is still dominated by solar data, most importantly by the CC/NC solar neutrino rate measured by SNO [8]. Moreover, as evident from Fig. 11 solar data fixes the octant of $\theta_{12}$, thanks to the MSW mechanism [9, 10] due to matter effects inside the sun, whereas the small matter effect in KamLAND cannot break the symmetry between the first and second $\theta_{12}$ octants.

We find a similar complementarity also in the determination of the atmospheric oscillation parameters, see middle panel in Fig. 1] In this case the $\Delta \Delta m_{31}^{2}$ jdetermination is dominated by data from the MINOS long-baseline $v_{\mu}$ disappearance experiment, which by now largely supersedes the pioneering $\mathrm{K} 2 \mathrm{~K}$ measurement [11], although in the global analysis the latter still contributes slightly to the lower bound on $j \Delta m_{31}^{2} j$ The determination of the mixing angle $\theta_{23}$ is dominated by atmospheric neutrino data from Super-Kamiokande [12], leading to a best fit point at maximal mixing 2 The sign of $\Delta m_{31}^{2}$ (i.e., the neutrino mass hierarchy) is undetermined by present data.

Similar to the case of the leading oscillation parameters, also the bound on $\theta_{13}$ emerges from an interplay of different data sets, as illustrated in right panel of Fig.11. An important contribution to the bound comes, of course, from the $\mathrm{CHOOZ}$ reactor experiment [15] combined with the determination of $j \Delta m_{31}^{2}$ jfrom atmospheric and long-baseline experiments. However, due to a complementarity of low and high energy solar data, as well as solar and KamLAND data also solar+KamLAND provide a non-trivial constraint on $\theta_{13}$, see e.g., [2, 3, 16]. We obtain at $90 \%$ CL $(3 \sigma)$ the following limits:

$$
\begin{array}{rll} 
& 8 \\
\sin ^{2} \theta_{13} & : 051(0.084) & \text { solar+KamLAND } \\
& : 028(0.059) & \text { CHOOZ+atm+LBL } \\
0.028(0050) & \text { global data }
\end{array}
$$

In the global analysis we find a slight weakening of the upper bound on $\sin ^{2} \theta_{13}$ due to the new data from 0.04 (see Ref. [17] or v5 of [3]) to 0.05 at $3 \sigma$. The reason for this is two-fold. First, the shift of the allowed range for $\dot{j} \Delta m_{31}^{2}$ jto lower values due to the new MINOS data implies a slightly weaker constraint on $\sin ^{2} \theta_{13}$ (cf. Fig. 1), and second, the combination of solar and new KamLAND data prefers a slightly non-zero value of $\sin ^{2} \theta_{13}$ which, though not statistically significant, also results in a weaker constraint in the global fit. Note also that sub-leading effects in atmospheric neutrino data have an impact on the bound on $\theta_{13}$ at that level, as discussed in Ref. [17].

\footnotetext{
${ }^{2}$ Small deviations from maximal mixing due to sub-leading threeflavour effects are discussed in Refs. [13, 14]. At present such deviations are not statistically significant.
} 

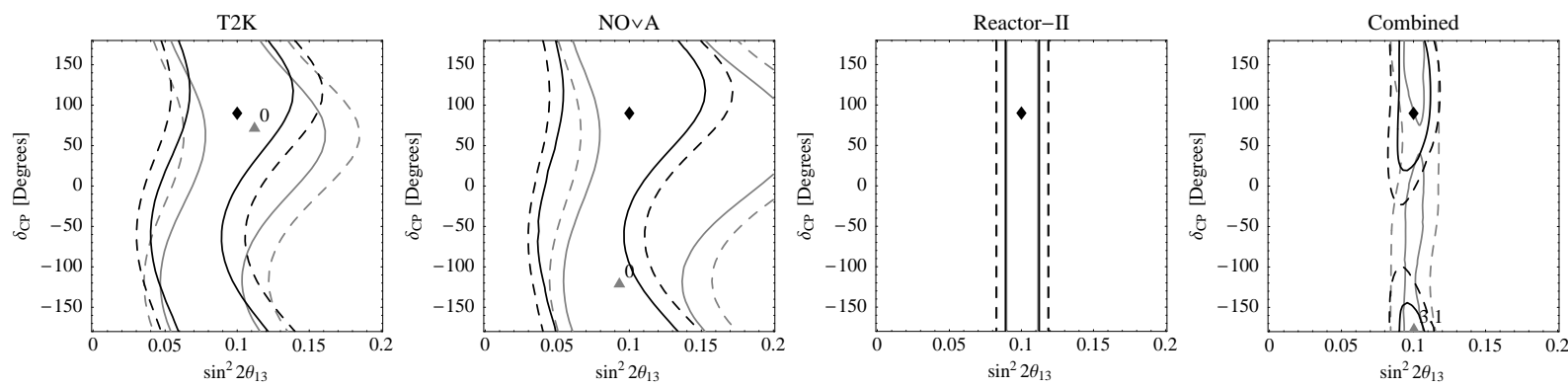

FIGURE 2. The $90 \% \mathrm{CL}$ (solid) and $3 \sigma$ (dashed) allowed regions ( 2 d.o.f.) in the $\sin ^{2} 2 \theta_{13}-\delta$ plane for the true values $\sin ^{2} 2 \theta_{13}=0: 1$ and $\delta=90$ for T2K, NOvA, a reactor experiment, and the combination. The black curves refer to the allowed regions for the normal mass hierarchy, whereas the gray curves refer to the inverted hierarchy. The best fits are marked as diamonds (normal hierarchy) and triangles (inverted hierarchy). For the latter, the $\Delta \chi^{2}$-value with respect to the best-fit point is also given [18].

\section{OUTLOOK FOR THE NEAR FUTURE}

In the following I try to give some outlook on developments in neutrino oscillations to be expected at a time scale of 5 to 10 years [18]. In this time-frame we expect results from a new generation of reactor experiments, Double-Chooz [19] and Daya-Bay [20], as well as the next generation of long-baseline superbeam experiments T2K [21] and NOvA [22].

The currently running MINOS experiment will improve further the determination of $j \Delta m_{31}^{2}$ jwith accumulating statistics. Once results on $v_{\mu}$ disappearance become available from the $\mathrm{T} 2 \mathrm{~K}$ and/or NOvA experiments a determination of this parameter at the level of a few percent at $2 \sigma$ will be obtained [18, 21, 22] (currently $12 \%$, cf. Tab. 11, and also $\sin ^{2} \theta_{23}$ is likely to be measured with a precision better than present atmospheric neutrino data.

Certainly the main goal of the upcoming experiments is the determination of $\theta_{13}$. Reactor experiments aim at this goal by exploring the disappearance of $\bar{v}_{e}$. The corresponding survival probability is given to very good accuracy by

$$
P_{e e}=1 \quad \sin ^{2} 2 \theta_{13} \sin ^{2} \frac{\Delta m_{31}^{2} L}{4 E_{v}}:
$$

This simple dependence shows that reactor experiments provide a clean measurement of $\sin ^{2} 2 \theta_{13}$, not affected by correlation or degeneracies with other unknown parameters [23]. The main issue in such an experiment are statistical and systematical errors, where the latter are going to be addressed by comparing data for near and far detectors. In contrast, the superbeam experiments look for the appearance of $v_{e}$ from a beam consisting initially mainly of $v_{\mu}$. At leading order in the small parameters $\sin 2 \theta_{13}$ and $\tilde{\alpha} \quad \sin 2 \theta_{12} \Delta m_{21}^{2} L=4 E_{v}$ the relevant oscil- lation probability (in vacuum, for simplicity) is

$$
\begin{aligned}
P_{\mu e} & =\sin ^{2} 2 \theta_{13} \sin ^{2} \theta_{23} \sin ^{2} \Delta+\tilde{\alpha}^{2} \cos ^{2} \theta_{23} \\
& +\sin 2 \theta_{13} \sin 2 \theta_{23} \tilde{\alpha} \sin \Delta \cos \left(\begin{array}{ll}
\Delta & \delta
\end{array}\right) ;
\end{aligned}
$$

where $\Delta \quad \Delta m_{31}^{2} L=4 E_{v}$, and '+' (' ') holds for neutrinos (anti-neutrinos). This expression shows that there is a complicated correlation of $\sin ^{2} 2 \theta_{13}$ with other parameters, especially with the CP phase $\delta$. This effect is illustrated in Fig. 22 where the allowed region in the plane of $\sin ^{2} 2 \theta_{13}$ and $\delta$ is shown for T2K, NOvA, a reactor experiment, and the combination, assuming an input value of $\sin ^{2} 2 \theta_{13}=0: 1$ and $\delta=90$. For the superbeams the allowed regions show a typical ' $S$ '-shape, reflecting the trigonometric dependence of the probability on $\delta$. Furthermore, solutions with the wrong mass hierarchy (gray curves in the figure) introduce another ambiguity in the interpretation. On the other hand, the figure shows that a reactor experiment can determine $\sin ^{2} 2 \theta_{13}$ unambiguously. The right most panel illustrates the situation which could emerge from the global analysis of these experiments: A relative good determination of $\theta_{13}$, some information on $\delta$ (though CP violation cannot be established), which however is largely corrupted by the ambiguity in the mass hierarchy, which cannot be resolved in this particular example ( $\Delta \chi^{2}$ of the wrong hierarchy is only 3.1 in the global analysis).

Fig. 3 shows the evolution of the $\theta_{13}$ discovery reach as a function of time, where of course a significant uncertainty is associated with the horizontal axis. The complementarity of beam and reactor experiments is also visible in that figure: The wide bands for the beam experiments follow from the impact of the (unknown) true value of $\delta$, which could be in favor for discovering a non-zero value of $\theta_{13}$ or not. In contrast, reactor experiments do not depend on the CP-phase and their reach is just determined by statistics and systematics.

Clearly, with the next generation of experiments we are entering the era of precision measurements, at the 


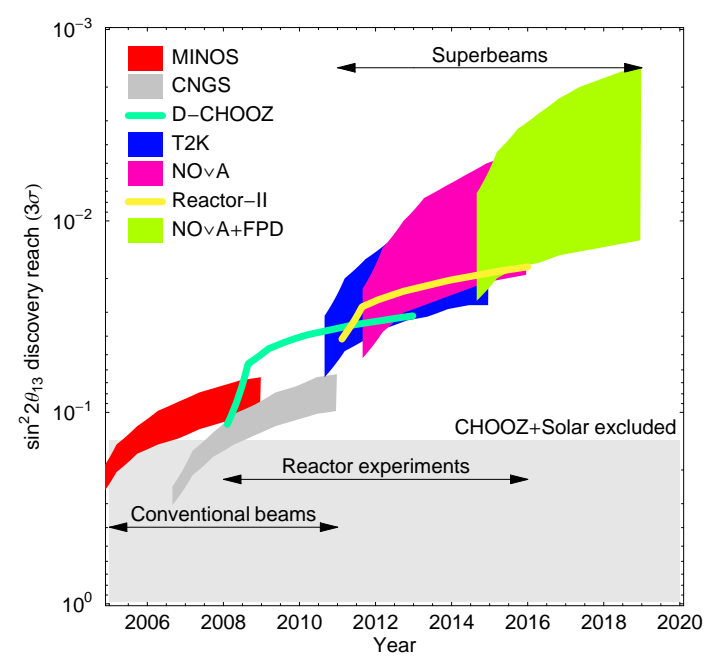

FIGURE 3. Evolution of the $3 \sigma$ discovery potential of a nonzero value of $\theta_{13}$ of upcoming experiments. Figure from [24].

level of $1 \%$. The are many ideas on how to go beyond this level, performing high precision measurements addressing questions like leptonic $\mathrm{CP}$ violation or the type of the neutrino mass hierarchy, among them high-intensity superbeams, beta beams or neutrino factories. These options have been discussed at this conference.

\section{LSND AND MINIBOONE RESULTS}

Reconciling the LSND evidence [25] for $\bar{v}_{\mu} ! \bar{v}_{e}$ oscillations with the global neutrino data reporting evidence and bounds on oscillations remains a long-standing problem for neutrino phenomenology. Recently the MiniBooNE experiment [26, 27] added more information to this question. This experiment searches for $v_{\mu} ! v_{e}$ appearance with a very similar $L=E_{v}$ range as LSND. No evidence for oscillations is found and the results are inconsistent with a two-neutrino oscillation interpretation of LSND at 98\% CL [27]. The exclusion contour from MiniBooNE is shown in Fig. 4 in comparison to the LSND allowed region and the previous bound from the KARMEN experiment [28], all in the framework of 2flavour oscillations.

The standard "solution" to the LSND problem is to introduce one or more sterile neutrinos at the $\mathrm{eV}$ scale in order to provide the required mass-squared difference to accommodate the LSND signal in addition to "solar" and "atmospheric" oscillations. However, in such schemes there is a sever tension between the LSND signal and short-baseline disappearance experiments, most importantly Bugey [29] and CDHS [30], with some contribution also from atmospheric neutrino data [31]. I report

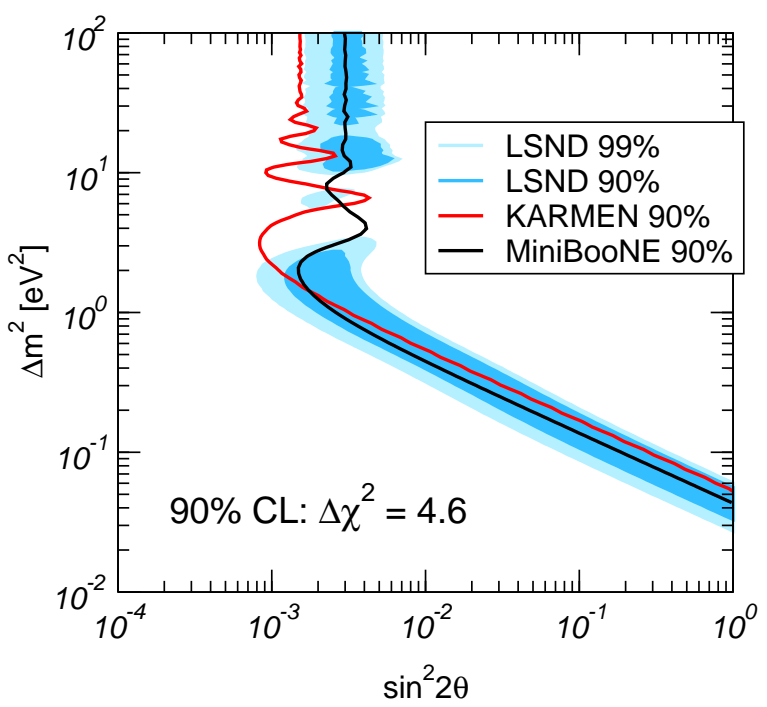

FIGURE 4. Exclusion contours at $90 \%$ (2 d.o.f.) for MiniBooNE and KARMEN compared to the LSND allowed region.

here the results from [32], where a global analysis including the MiniBooNE results has been performed in schemes with one, two and three sterile neutrinos (see also [33]).

Four-neutrino oscillations within so-called $(3+1)$ schemes have been only marginally allowed before the recent MiniBooNE results (see, e.g., Refs. [34, 3, 35]), and become even more disfavored with the new data, at the level of $4 \sigma$ [32]. Five-neutrino oscillations in $(3+2)$ schemes [35] allow for the possibility of $\mathrm{CP}$ violation in short-baseline oscillations [36]. Using the fact that in LSND the signal is in anti-neutrinos, whereas present MiniBooNE data is based on neutrinos, these two experiments become fully compatible in $(3+2)$ schemes [32]. However, in the global analysis the tension between appearance and disappearance experiments remains unexplained. This problem is illustrated in Fig. 5 where sections through the allowed regions in the parameter space for appearance and disappearance experiments are shown. An opposite trend is clearly visible: while appearance data require non-zero values for the mixing of $v_{e}$ and $v_{\mu}$ with the $\mathrm{eV}$-scale mass states 4 and 5 in order to explain LSND, disappearance data provide an upper bound on these mixing. The allowed regions touch each other at $\Delta \chi^{2}=9 \cdot 3$, and a consistency test between these two data samples yields a probability of only $0: 18 \%$, i.e., these models can be considered as disfavoured at the $3 \sigma$ level. Furthermore, when moving from 4 neutrinos to 5 neutrinos the fit improves only by 6.1 units in $\chi^{2}$ by introducing 4 more parameters, showing that in $(3+2)$ schemes the tension in the fit remains a sever problem. This is even true in the case of three sterile neutrinos, since adding one more neutrino to $(3+2)$ cannot improve 


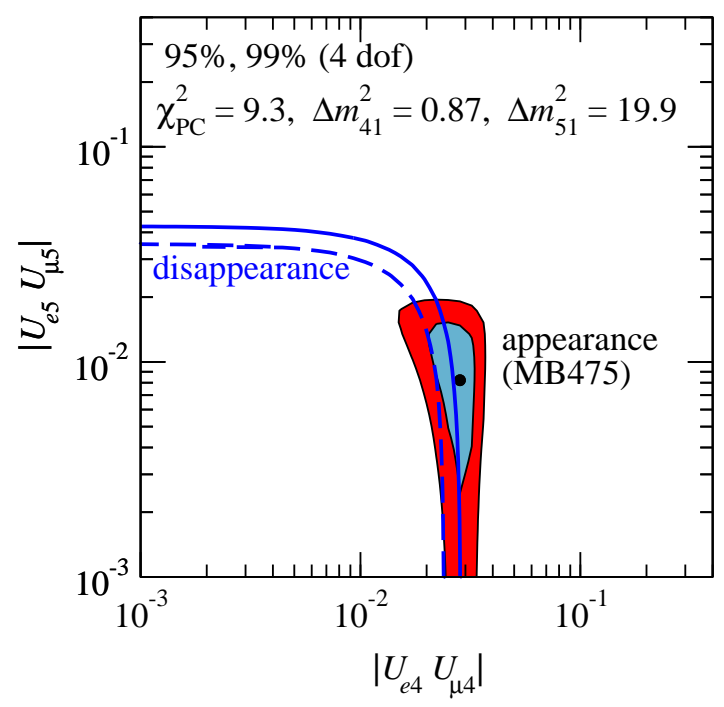

FIGURE 5. Section of the 4-dimensional volumes allowed at 95\% and $99 \%$ CL in the $(3+2)$ scheme from SBL appearance and disappearance experiments in the space of the parameters in common to these two data sets. The values of $\Delta m_{41}^{2}$ and $\Delta m_{51}^{2}$ of the displayed sections correspond to the point in parameter space where the two allowed regions touch each other (at a $\left.\Delta \chi^{2}=9: 3\right)$.

the situation [32] 3

In view of this somewhat ambiguous situation I comment in the following on the impact of sterile neutrinos (with masses and mixing relevant for LSND) for future neutrino oscillation experiments. As discussed in the previous section the typical $\theta_{13}$-sensitivity of the next generation of experiments (Double-Chooz, T2K, NOvA) is $\sin ^{2} 2 \theta_{13} \& 1 \%$, cf. Fig. 3 This should be compared to the size of the appearance probability observed in LSND: $P_{\text {LSND }} 0.26 \%$. Hence, if $\theta_{13}$ is large enough to be found in those experiments sterile neutrinos may introduce some sub-leading effect, but their presence cannot be confused with a non-zero $\theta_{13}$. Nevertheless, I argue that it could be worth to look for sterile neutrino effects in the next generation of experiments. They would introduce (mostly energy averaged) effects, which could be visible as disappearance signals in the near detectors of these experiments. This has been discussed in [38] for the Double-Chooz experiment, but also the near detectors at superbeam experiments should be explored 4

However, for the subsequent generation of oscillation experiments aiming at sub-percent level precision to test $\mathrm{CP}$ violation and the neutrino mass hierarchy, the ques-

\footnotetext{
${ }^{3}$ In Ref. [37] I have pointed out that an exotic sterile neutrino with energy dependent mass or mixing can resolve these tensions.

${ }^{4}$ An interesting effect of $(3+2)$ schemes has been pointed out recently for high energy atmospheric neutrinos in neutrino telescopes [39].
}

tion of LSND sterile neutrinos is highly relevant [40, 41]. They will lead to a miss-interpretation or (in the best case) to an inconsistency in the results. If eV scale steriles exist with mixing relevant for LSND the optimization in terms of baseline and $E_{v}$ of high precision experiments has to be significantly changed. Therefore, I argue that it is important to settle this question at high significance before decisions on high precision oscillation facilities are taken.

Acknowledgment. The author acknowledges support from the BENE network.

\section{REFERENCES}

1. T. Kajita (2007), these proceedings.

2. M. Maltoni, T. Schwetz, M. A. Tortola, and J. W. F. Valle, Phys. Rev. D68, 113010 (2003), hep-ph/0309130.

3. M. Maltoni, T. Schwetz, M. A. Tortola, and J. W. F. Valle, New J. Phys. 6, 122 (2004), hep-ph/ 0405172 v6.

4. MINOS (2007), arXiv:0708.1495 [hep-ex]

5. Z. Pavlovic (2007), these proceedings.

6. I. Shimizu (2007), talk at the 10th International Conference on Topics in Astroparticle and Underground Physics, TAUP2007.

7. T. Araki, et al., Phys. Rev. Lett. 94, 081801 (2005), hep-ex/0406035

8. B. Aharmim, et al., Phys. Rev. C72, 055502 (2005), nucl-ex/0502021.

9. L. Wolfenstein, Phys. Rev. D17, 2369 (1978).

10. S. P. Mikheev, and A. Y. Smirnov, Sov. J. Nucl. Phys. 42 , 913-917 (1985).

11. E. Aliu, et al., Phys. Rev. Lett. 94, 081802 (2005), hep-ex/0411038

12. Y. Ashie, et al., Phys. Rev. D71, 112005 (2005), hep-ex/0501064

13. G. L. Fogli, E. Lisi, A. Marrone, A. Palazzo, and A. M. Rotunno, Prog. Part. Nucl. Phys. 57, 71-78 (2006).

14. M. C. Gonzalez-Garcia, and M. Maltoni (2007), arXiv:0704.1800[hep-ph]

15. M. Apollonio, et al., Eur. Phys. J. C27, 331-374 (2003), hep-ex/0301017

16. S. Goswami, and A. Y. Smirnov, Phys. Rev. D72, 053011 (2005), hep-ph/0411359

17. T. Schwetz, Phys. Scripta T127, 1-5 (2006), hep-ph/0606060

18. P. Huber, M. Lindner, M. Rolinec, T. Schwetz, and W. Winter, Phys. Rev. D70, 073014 (2004), hep-ph/0403068

19. T. Kawasaki (2007), these proceedings.

20. C. Mauger (2007), these proceedings.

21. T. Nakadaira (2007), these proceedings.

22. A. Norman (2007), these proceedings.

23. P. Huber, M. Lindner, T. Schwetz, and W. Winter, Nucl. Phys. B665, 487-519 (2003), hep-ph/0303232

24. M. G. Albrow, et al. (2005), hep-ex/0509019

25. A. Aguilar, et al., Phys. Rev. D64, 112007 (2001), hep-ex/0104049

26. C. Polly (2007), these proceedings. 
27. A. A. Aguilar-Arevalo, et al., Phys. Rev. Lett. 98, 231801 (2007), arXiv: 0704.1500 [hep-ex]

28. B. Armbruster, et al., Phys. Rev. D65, 112001 (2002), hep-ex/0203021

29. Y. Declais, et al., Nucl. Phys. B434, 503-534 (1995).

30. F. Dydak, et al., Phys. Lett. B134, 281 (1984).

31. S. M. Bilenky, C. Giunti, W. Grimus, and T. Schwetz, Phys. Rev. D60, 073007 (1999), hep-ph/9903454

32. M. Maltoni, and T. Schwetz, Phys. Rev. D, to appear (2007), arXiv:0705.0107 [hep-ph]

33. G. Karagiorgi (2007), these proceedings.

34. M. Maltoni, T. Schwetz, M. A. Tortola, and J. W. F. Valle, Nucl. Phys. B643, 321-338 (2002), hep-ph/0207157

35. M. Sorel, J. M. Conrad, and M. Shaevitz, Phys. Rev. D70, 073004 (2004), hep-ph/0305255

36. G. Karagiorgi, et al., Phys. Rev. D75, 013011 (2007), hep-ph/0609177

37. T. Schwetz (2007), arXiv:0710.2985 [hep-ph]

38. A. Bandyopadhyay, and S. Choubey (2007), arXiv:0707.2481[hep-ph].

39. S. Choubey (2007), arXiv:0709.1937 [hep-ph]

40. A. Donini, M. Lusignoli, and D. Meloni, Nucl. Phys. B624, 405-422 (2002), hep-ph/0107231.

41. A. Dighe, and S. Ray (2007), arXiv:0709.0383 [hep-ph] 